\title{
HeI Photoelectron Spectra and Gas-phase Electronic Structures of End-functionalized [3]- and [5]-Ladderanes
}

Tomislav Friščic ${ }^{1}$,Leo Klasinc ${ }^{2 *}$, Branka Kovač $^{2}$ and Leonard R.MacGillivray ${ }^{1 *}$

${ }^{1}$ Department of Chemistry, University of Iowa, Iowa City, IA 52242-1294, USA

${ }^{2}$ Department of Physical Chemistry, The Rudjer Bošković Institute, HR-10002 Zagreb, Croatia

\section{Supplementary material}

Figure S1. Diagram of molecule: a) 1 and b) 2, with the numbering scheme used in computations shown.

Table S1. Experimental (X-ray) and calculated (DFT) bond lengths, differences between calculated and experimental bond lengths and squares of the differences, $r_{\mathrm{X}-\text { ray }}, r_{\mathrm{DFT}}, r_{\mathrm{DFT}^{-}}$ $r_{\mathrm{X} \text {-ray }}(\AA)$ and $\left(r_{\mathrm{DFT}}-r_{\mathrm{X} \text {-ray }}\right)^{2}\left(\AA^{2}\right)$ for compound $\mathbf{1}$.

Table S2. Experimental (X-ray) and calculated (B3LYP) distances between pairs of atoms, differences between calculated and experimental bond lengths and squares of the differences, $r_{\mathrm{X} \text {-ray }}, r_{\mathrm{B} 3 \mathrm{LYP}}, r_{\mathrm{B} 3 \mathrm{LYP}}{ }^{-} r_{\mathrm{X} \text {-ray }}(\AA)$ and $\left(r_{\mathrm{B} 3 \mathrm{LYP}}-r_{\mathrm{X} \text {-ray }}\right)^{2}\left(\AA^{2}\right)$ for compound 2.

Table S3. Coordinates of optimized geometries [x, y, z in Á] of 1, 2, [3]-ladderane,[3]ladderene, and [3]-ladderdiene hydrocarbons 
a)<smiles>[CH]C1CCNCC1</smiles>
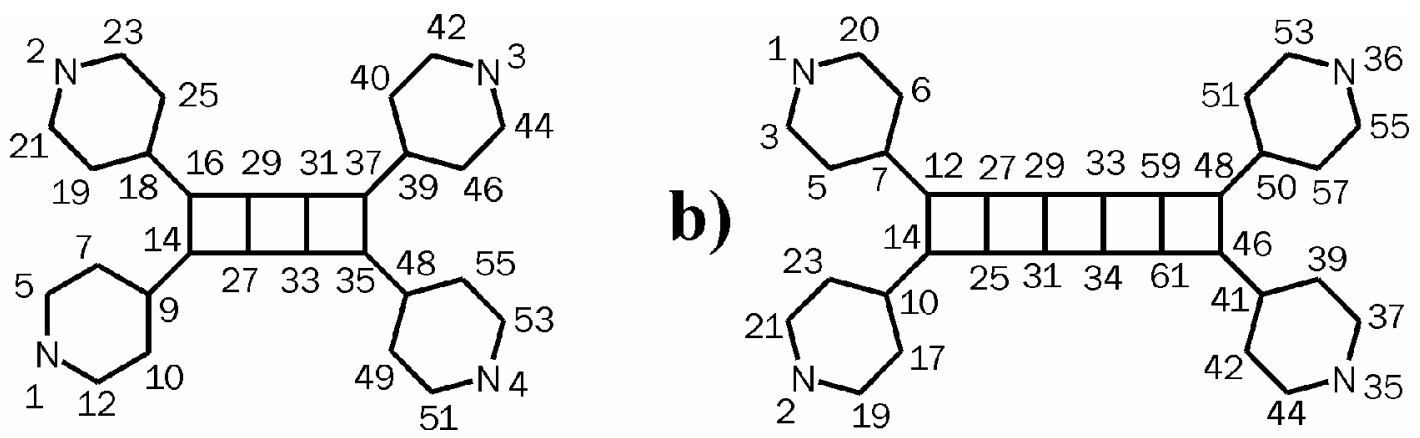

Figure S1. Diagram of molecule: a) 1 and b) 2, with the numbering scheme used in computations shown.

Table S1. Experimental (X-ray) and calculated (DFT) bond lengths, differences between calculated and experimental bond lengths and squares of the differences, $r_{\mathrm{X} \text {-ray }}, r_{\mathrm{DFT}}, r_{\mathrm{DFT}}-$ $r_{\mathrm{X} \text {-ray }}(\AA)$ and $\left(r_{\mathrm{DFT}}-r_{\mathrm{X} \text {-ray }}\right)^{2}\left(\AA^{2}\right)$ for compound $\mathbf{1}$.

\begin{tabular}{lllll}
\hline Atom numbers & $r_{\text {X-Ray }}$ & $r_{\text {DFT }}$ & $r_{\text {DFT }}-r_{\text {X-ray }}$ & $\left(r_{\text {DFT }}-r_{\text {X-ray }}\right)^{2}$ \\
\hline$(1,5)$ & 1.3254 & 1.3509 & 0.0255 & 0.00065025 \\
$(1,12)$ & 1.3359 & 1.3525 & 0.0166 & 0.00027556 \\
$(2,21)$ & 1.3348 & 1.3509 & 0.0161 & 0.00025921 \\
$(2,23)$ & 1.3417 & 1.3525 & 0.0108 & 0.00011664 \\
$(3,42)$ & 1.3352 & 1.3509 & 0.0157 & 0.00024649 \\
$(3,44)$ & 1.336 & 1.3525 & 0.0165 & 0.00027225 \\
$(4,51)$ & 1.3355 & 1.3525 & 0.017 & 0.000289 \\
$(4,53)$ & 1.3353 & 1.3509 & 0.0156 & 0.00024336 \\
$(5,7)$ & 1.383 & 1.3979 & 0.0149 & 0.00022201 \\
$(7,9)$ & 1.3942 & 1.4053 & 0.0111 & 0.00012321 \\
$(9,10)$ & 1.386 & 1.4068 & 0.0208 & 0.00043264 \\
$(9,14)$ & 1.498 & 1.508 & 0.01 & 0.0001 \\
$(10,12)$ & 1.3758 & 1.3959 & 0.0201 & 0.00040401 \\
$(14,16)$ & 1.5929 & 1.6178 & 0.0249 & 0.00062001 \\
$(14,27)$ & 1.5316 & 1.5609 & 0.0293 & 0.00085849 \\
$(16,18)$ & 1.5019 & 1.5074 & 0.0055 & 0.00003025 \\
$(16,29$ & 1.5528 & 1.5629 & 0.0101 & 0.00010201 \\
$(18,19)$ & 1.3881 & 1.4053 & 0.0172 & 0.00029584 \\
$(18,25)$ & 1.3802 & 1.4069 & 0.0267 & 0.00071289 \\
$(19,21)$ & 1.3745 & 1.3978 & 0.0233 & 0.00054289 \\
$(23,25)$ & 1.3786 & 1.396 & 0.0174 & 0.00030276 \\
$(27,29)$ & 1.5649 & 1.5885 & 0.0236 & 0.00055696 \\
$(27,33)$ & 1.547 & 1.5556 & 0.0086 & 0.00007396 \\
$(29,31)$ & 1.5117 & 1.5521 & 0.0404 & 0.00163216 \\
$(31,33)$ & 1.5748 & 1.5885 & 0.0137 & 0.00018769 \\
$(31,37)$ & 1.5422 & 1.5628 & 0.0206 & 0.00042436 \\
$(33,35)$ & 1.5297 & 1.561 & 0.0313 & 0.00097969 \\
$(35,37)$ & 1.5934 & 1.6178 & 0.0244 & 0.00059536 \\
\hline & & & &
\end{tabular}


Table S1 (contd.). Experimental (X-ray) and calculated (DFT) bond lengths, differences between calculated and experimental bond lengths and squares of the differences, $r_{\mathrm{X} \text {-ray }}$, $r_{\mathrm{DFT}}, r_{\mathrm{DFT}}-r_{\mathrm{X} \text {-ray }}(\AA)$ and $\left(r_{\mathrm{DFT}}-r_{\mathrm{X} \text {-ray }}\right)^{2}\left(\AA^{2}\right)$ for compound $\mathbf{1}$.

\begin{tabular}{lllll}
\hline Atom numbers & $r_{\text {X-Ray }}$ & $r_{\text {DFT }}$ & $r_{\text {DFT }}-r_{\text {X-ray }}$ & $\left(r_{\text {DFT }}-r_{\text {X-ray }}\right)^{2}$ \\
\hline$(35,48)$ & 1.5042 & 1.508 & 0.0038 & 0.00001444 \\
$(37,39)$ & 1.4967 & 1.5074 & 0.0107 & 0.00011449 \\
$(39,40)$ & 1.398 & 1.4053 & 0.0073 & 0.00005329 \\
$(39,46)$ & 1.3858 & 1.407 & 0.0212 & 0.00044944 \\
$(40,42)$ & 1.372 & 1.3978 & 0.0258 & 0.00066564 \\
$(44,46)$ & 1.378 & 1.396 & 0.018 & 0.000324 \\
$(48,49)$ & 1.3781 & 1.4068 & 0.0287 & 0.00082369 \\
$(48,55)$ & 1.3915 & 1.4053 & 0.0138 & 0.00019044 \\
$(49,51)$ & 1.3804 & 1.3959 & 0.0155 & 0.00024025 \\
$(53,55)$ & 1.3741 & 1.3979 & 0.0238 & 0.00056644 \\
\hline
\end{tabular}

Table S2. Experimental (X-ray) and calculated (B3LYP) distances between pairs of atoms, differences between calculated and experimental bond lengths and squares of the differences, $r_{\mathrm{X} \text {-ray }}, r_{\mathrm{B} 3 \mathrm{LYP}}, r_{\mathrm{B} 3 \mathrm{LYP}} r_{\mathrm{X} \text {-ray }}(\AA)$ and $\left(r_{\mathrm{B} 3 \mathrm{LYP}} r_{\mathrm{X} \text {-ray }}\right)^{2}\left(\AA^{2}\right)$ for compound 2.

\begin{tabular}{lllll}
\hline Atom numbers & $r_{\mathrm{X} \text {-Ray }}$ & $r_{\mathrm{B} 3 \mathrm{LYP}}$ & $r_{\mathrm{B} 3 \mathrm{LYP}-r_{\mathrm{X} \text {-ray }}}$ & $\left(r_{\mathrm{B} 3 \mathrm{LY}-}-r_{\mathrm{X} \text {-ray }}\right)^{2}$ \\
\hline$(1,3)$ & 1.326 & 1.351 & 0.025 & 0.000625 \\
$(1,10)$ & 1.337 & 1.3525 & 0.0155 & 0.00024025 \\
$(2,19)$ & 1.329 & 1.351 & 0.022 & 0.000484 \\
$(2,21)$ & 1.323 & 1.3525 & 0.0295 & 0.00087025 \\
$(3,5)$ & 1.378 & 1.3979 & 0.0199 & 0.00039601 \\
$(5,7)$ & 1.386 & 1.4054 & 0.0194 & 0.00037636 \\
$(7,8)$ & 1.379 & 1.407 & 0.028 & 0.000784 \\
$(7,12)$ & 1.495 & 1.5076 & 0.0126 & 0.00015876 \\
$(8,10)$ & 1.371 & 1.3959 & 0.0249 & 0.00062001 \\
$(12,14)$ & 1.585 & 1.6184 & 0.0334 & 0.00111556 \\
$(12,27)$ & 1.55 & 1.5616 & 0.0116 & 0.00013456 \\
$(14,16)$ & 1.496 & 1.5076 & 0.0116 & 0.00013456 \\
$(14,25)$ & 1.538 & 1.5616 & 0.0236 & 0.00055696 \\
$(16,17)$ & 1.392 & 1.4054 & 0.0134 & 0.00017956 \\
$(16,23)$ & 1.388 & 1.407 & 0.019 & 0.000361 \\
$(17,19)$ & 1.362 & 1.3979 & 0.0359 & 0.00128881 \\
$(21,23)$ & 1.394 & 1.3959 & 0.0019 & 0.00000361 \\
$(25,27)$ & 1.567 & 1.5853 & 0.0183 & 0.00033489 \\
$(25,31)$ & 1.531 & 1.5518 & 0.0208 & 0.00043264 \\
$(27,29)$ & 1.522 & 1.5517 & 0.0297 & 0.00088209 \\
$(29,31)$ & 1.586 & 1.586 & 0 & 0 \\
$(29,33)$ & 1.527 & 1.5492 & 0.0222 & 0.00049284 \\
$(31,34)$ & 1.527 & 1.5492 & 0.0222 & 0.00049284 \\
\hline
\end{tabular}


Table S2 (contd.). Experimental (X-ray) and calculated (B3LYP) distances between pairs of atoms, differences between calculated and experimental bond lengths and squares of the differences, $r_{\mathrm{X} \text {-ray }}, r_{\mathrm{B} 3 \mathrm{LYP}}, r_{\mathrm{B} 3 \mathrm{LYP}}-r_{\mathrm{X} \text {-ray }}(\AA)$ and $\left(r_{\mathrm{B} 3 \mathrm{LYP}}-r_{\mathrm{X} \text {-ray }}\right)^{2}\left(\AA^{2}\right)$ for compound $\mathbf{2}$.

\begin{tabular}{lllll}
\hline Atom numbers & $r_{\mathrm{X} \text {-Ray }}$ & $r_{\mathrm{B} 3 \mathrm{LYP}}$ & $r_{\mathrm{B} 3 \mathrm{LYP}-} r_{\mathrm{X} \text {-ray }}$ & $\left(r_{\mathrm{B} 3 \mathrm{LYP}}-r_{\mathrm{X} \text {-ray }}\right)^{2}$ \\
\hline$(33,59)$ & 1.531 & 1.5517 & 0.0207 & 0.00042849 \\
$(33,34)$ & 1.586 & 1.586 & 0 & 0 \\
$(34,61)$ & 1.522 & 1.5517 & 0.0297 & 0.00088209 \\
$(35,37)$ & 1.326 & 1.351 & 0.025 & 0.000625 \\
$(35,44)$ & 1.337 & 1.3525 & 0.0155 & 0.00024025 \\
$(36,53)$ & 1.329 & 1.351 & 0.022 & 0.000484 \\
$(36,55)$ & 1.323 & 1.3525 & 0.0295 & 0.00087025 \\
$(37,39)$ & 1.378 & 1.3979 & 0.0199 & 0.00039601 \\
$(39,41)$ & 1.386 & 1.4054 & 0.0194 & 0.00037636 \\
$(41,42)$ & 1.379 & 1.407 & 0.028 & 0.000784 \\
$(41,46)$ & 1.495 & 1.5076 & 0.0126 & 0.00015876 \\
$(42,44)$ & 1.371 & 1.3959 & 0.0249 & 0.00062001 \\
$(46,48)$ & 1.585 & 1.6184 & 0.0334 & 0.00111556 \\
$(46,61)$ & 1.55 & 1.5616 & 0.0116 & 0.00013456 \\
$(48,50)$ & 1.496 & 1.5076 & 0.0116 & 0.00013456 \\
$(48,59)$ & 1.538 & 1.5616 & 0.0236 & 0.00055696 \\
$(50,51)$ & 1.392 & 1.4054 & 0.0134 & 0.00017956 \\
$(50,57)$ & 1.388 & 1.407 & 0.019 & 0.000361 \\
$(51,53)$ & 1.362 & 1.3978 & 0.0358 & 0.00128164 \\
$(55,57)$ & 1.394 & 1.3959 & 0.0019 & 0.00000361 \\
$(59,61)$ & 1.567 & 1.5853 & 0.0183 & 0.00033489 \\
\hline
\end{tabular}


Table S3. Coordinates of optimized geometries [x, y, z in Á] of 1, 2, [3]-ladderane,[3]ladderene and [3]-ladderdiene hydrocarbons

\section{Coordinates of $\mathbf{1}$}

\begin{tabular}{|c|c|c|c|}
\hline C & 1.89803230 & -0.85697945 & -0.65148527 \\
\hline $\mathrm{C}$ & 1.87935406 & 0.76001667 & -0.69817385 \\
\hline $\mathrm{C}$ & 0.70233465 & 0.76295054 & 0.32994870 \\
\hline $\mathrm{C}$ & 0.69708166 & -0.82548115 & 0.34514685 \\
\hline $\mathrm{C}$ & -0.70236958 & 0.76283989 & -0.33014280 \\
\hline $\mathrm{C}$ & -0.69704601 & -0.82560558 & -0.34500065 \\
\hline $\mathrm{C}$ & -1.87934955 & 0.76004794 & 0.69803659 \\
\hline $\mathrm{C}$ & -1.89802614 & -0.85696650 & 0.65158051 \\
\hline $\mathrm{C}$ & -3.17269189 & -1.53341199 & 0.21393348 \\
\hline $\mathrm{C}$ & -3.14614612 & 1.50037381 & 0.35269836 \\
\hline C & 3.17270936 & -1.53340691 & -0.21384712 \\
\hline C & 3.14613441 & 1.50037188 & -0.35280668 \\
\hline C & -4.07304824 & -2.01832090 & 1.18005259 \\
\hline $\mathrm{C}$ & -5.25977038 & -2.63377824 & 0.77826175 \\
\hline $\mathrm{N}$ & -5.60356627 & -2.79957162 & -0.51924398 \\
\hline $\mathrm{C}$ & -4.74188801 & -2.33424787 & -1.44972404 \\
\hline C & -3.53526650 & -1.70340218 & -1.13308929 \\
\hline $\mathrm{C}$ & -3.55523481 & 1.75828791 & -0.96678661 \\
\hline $\mathrm{C}$ & -4.75049173 & 2.44511541 & -1.19802520 \\
\hline $\mathrm{N}$ & -5.55786265 & 2.88539848 & -0.20841891 \\
\hline $\mathrm{C}$ & -5.16910993 & 2.63576430 & 1.06268167 \\
\hline C & -3.98992888 & 1.95964786 & 1.38060536 \\
\hline $\mathrm{C}$ & 3.53540165 & -1.70315131 & 1.13317779 \\
\hline $\mathrm{C}$ & 4.74202242 & -2.33398796 & 1.44982301 \\
\hline $\mathrm{N}$ & 5.60359341 & -2.79952873 & 0.5193490 \\
\hline $\mathrm{C}$ & 5.25968731 & -2.63396663 & -0.77815445 \\
\hline $\mathrm{C}$ & 4.07295364 & -2.01853484 & -1.17995650 \\
\hline $\mathrm{C}$ & 3.99004338 & 1.95945587 & -1.38069216 \\
\hline C & 5.16921102 & 2.63559291 & -1.06274047 \\
\hline $\mathrm{N}$ & 5.55782826 & 2.88541343 & 0.20835857 \\
\hline C & 4.75032812 & 2.44531281 & 1.1979487 \\
\hline $\mathrm{C}$ & 3.55508012 & 1.75848765 & 0.96668335 \\
\hline $\mathrm{H}$ & 1.61622935 & -1.26534618 & -1.62877889 \\
\hline $\mathrm{H}$ & 1.55197864 & 1.09503381 & -1.68910634 \\
\hline $\mathrm{H}$ & 0.82959251 & 1.38559545 & 1.21796971 \\
\hline $\mathrm{H}$ & 0.79357326 & -1.43235987 & 1.24787470 \\
\hline $\mathrm{H}$ & -0.82970607 & 1.38528519 & -1.21829114 \\
\hline $\mathrm{H}$ & -0.79345357 & -1.43268862 & -1.24760025 \\
\hline $\mathrm{H}$ & -1.55187806 & 1.09516463 & 1.68890520 \\
\hline $\mathrm{H}$ & -1.61630496 & -1.26521967 & 1.62894456 \\
\hline $\mathrm{H}$ & -3.84535012 & -1.92261288 & 2.23733700 \\
\hline $\mathrm{H}$ & -5.96355311 & -3.01587653 & 1.51031746 \\
\hline $\mathrm{H}$ & -5.03608774 & -2.47733851 & -2.48437279 \\
\hline $\mathrm{H}$ & -2.89233402 & -1.35708256 & -1.93415029 \\
\hline $\mathrm{H}$ & -2.95705595 & 1.43681305 & -1.81151956 \\
\hline $\mathrm{H}$ & -5.08019610 & 2.65585331 & -2.21012193 \\
\hline $\mathrm{H}$ & -5.82933725 & 2.99952379 & 1.84309264 \\
\hline $\mathrm{H}$ & -3.72383463 & 1.79784575 & 2.42080992 \\
\hline $\mathrm{H}$ & 2.89255086 & -1.35664402 & 1.93422306 \\
\hline
\end{tabular}




$\mathrm{H}$
$\mathrm{H}$
$\mathrm{H}$
$\mathrm{H}$
$\mathrm{H}$
$\mathrm{H}$
$\mathrm{H}$

5.03631068

5.96339161

3. 84516092

3.72406441

5.82953649

5.07992702

2. 95678912
$-2.47690297$

- 3.01622529

$-1.92302351$

1.79750042

2.99919870

2. 65621141

1. 43717068
2.48447058

$-1.51020214$

$-2.23723825$

$-2.42090197$

$-1.84314083$

2. 21004566

1. 81139857

\section{Coordinates of $\mathbf{2}$}

$\mathrm{x}$

$$
\begin{aligned}
& -6.85638224 \\
& -6.85640536 \\
& -5.98878248 \\
& -6.26564088 \\
& -4.79714681 \\
& -4.14799439 \\
& -4.45674439 \\
& -5.36328222 \\
& -5.15231976 \\
& -6.53385384 \\
& -7.24178753 \\
& -3.19957187 \\
& -2.92862702 \\
& -3.19984974 \\
& -2.92979296 \\
& -4.45694548 \\
& -4.79711474 \\
& -4.14784082 \\
& -5.98866178 \\
& -6.26534607 \\
& -6.53411170 \\
& -7.24215563 \\
& -5.36362561 \\
& -5.15284916 \\
& -1.97581277 \\
& -2.05709367 \\
& -1.97595598 \\
& -2.05772302 \\
& -0.60302783 \\
& -0.51807758 \\
& -0.60301814 \\
& -0.51824295 \\
& 0.60295604 \\
& 0.60307978 \\
& 6.85681875 \\
& 6.85597953 \\
& 5.98864596 \\
& 6.26511630 \\
& 4.79690725 \\
& 4.14728354 \\
& 4.45701305 \\
& 5.36414912 \\
& -
\end{aligned}
$$

y

2.84699549

$-2.84588316$

2.39577528

2.57503522

1.73585275

1.40406889

1.51839824

1.98888687

1.85713022

2.63612892

3.00756972

0.80862660

1.17931283

$-0.80979774$

$-1.18136415$

$-1.51872187$

$-1.73335623$

$-1.39982985$

$-2.39267414$

$-2.56976710$

$-2.63771468$

$-3.01071628$

$-1.99126929$

$-1.86172119$

$-0.79309859$

$-1.40526350$

0.79215836

1.40414423

0.80281233

1.42883268

$-0.80328620$

$-1.42879607$

0.80252605

$-0.80357522$

$-2.84580399$

2.84705352

$-2.39407074$

$-2.57230993$

$-1.73485969$

$-1.40259869$

$-1.51873072$

$-1.98974670$
Z
$-0.42903925$
$-0.43427907$
$-1.36112038$
$-2.39495597$
$-1.04726423$
$-1.84930108$
0.29883044
1. 26653226
2. 32345886
0.86742401
1.60104702
0.73299650
1.72825052
0.73255197
1.72771049
0.29678782
$-1.04982975$
$-1.85105266$
$-1.36526986$
$-2.39952505$
0.86267183
1.59539619
1. 26333840
2. 32057408
$-0.23699036$
$-1.13764524$
$-0.23710924$
$-1.13783644$
0.48591779
1.37926654
0.48638656
1.38010896
$-0.48646990$
$-0.48583655$
0.43315690
0.43015820
1.36445505
2.39857128
1.04949141
1.85095116
$-0.29694610$
$-1.26382863$ 


$\begin{array}{lrrr}\mathrm{H} & 5.15360761 & -1.85899898 & -2.32096363 \\ \mathrm{C} & 6.53478244 & -2.63620440 & -0.86363340 \\ \mathrm{H} & 7.24317503 & -3.00803211 & -1.59661687 \\ \mathrm{C} & 3.19977630 & -0.80978988 & -0.73230812 \\ \mathrm{H} & 2.92935754 & -1.18150388 & -1.72732066 \\ \mathrm{C} & 3.19963859 & 0.80863499 & -0.73323691 \\ \mathrm{H} & 2.92905686 & 1.17917273 & -1.72863754 \\ \mathrm{C} & 4.45667390 & 1.51838627 & -0.29867000 \\ \mathrm{C} & 4.79735770 & 1.73434339 & 1.04760510 \\ \mathrm{H} & 4.14855643 & 1.40129671 & 1.84940784 \\ \mathrm{C} & 5.98880810 & 2.39436389 & 1.36193485 \\ \mathrm{H} & 6.26588578 & 2.57247431 & 2.39590962 \\ \mathrm{C} & 6.53318840 & 2.63762026 & -0.86646603 \\ \mathrm{H} & 7.24077506 & 3.01022836 & -1.59983229 \\ \mathrm{C} & 5.36275807 & 1.99039841 & -1.26604283 \\ \mathrm{H} & 5.15155821 & 1.85984053 & -2.32307059 \\ \mathrm{C} & 1.97584660 & 0.79248722 & 0.23666308 \\ \mathrm{H} & 2.05744781 & 1.40500881 & 1.13704575 \\ \mathrm{C} & 1.97591211 & -0.79276863 & 0.23743641 \\ \mathrm{H} & 2.05735734 & -1.40439435 & 1.13843827 \\ \mathrm{H} & 0.51832515 & -1.42970742 & -1.37912535 \\ \mathrm{H} & 0.51798576 & 1.42794457 & -1.38023778\end{array}$

\section{Coordinates of [3]-ladderane hydrocarbon}

$\mathrm{x}$

$$
\begin{array}{r}
2.00133279 \\
0.58071222 \\
2.00140018 \\
-0.58073460 \\
-2.00138916 \\
0.58075606 \\
-0.58072985 \\
-2.00134661 \\
2.06508313 \\
2.77178776 \\
0.44734826 \\
2.77186903 \\
2.06526243 \\
-0.44733049 \\
-2.77179509 \\
-2.06532123 \\
0.44740767 \\
-0.44742778 \\
-2.77186025 \\
-2.06502958
\end{array}
$$

Y

0.78283820

0.79890928

$-0.78275445$

0.79896104

0.78279671

$-0.79901254$

$-0.79895665$

$-0.78279680$

1. 25918874

1. 21672507

1.40953889

$-1.21659329$

$-1.25904511$

1. 40968196

1.21657240

1. 25919610

$-1.40978198$

$-1.40960851$

$-1.21675174$

$-1.25903122$ $\mathrm{z}$

0.12846463

$-0.51365306$

0.12850617

0.51356592

$-0.12845106$

$-0.51354662$

0.51362816

$-0.12851688$

1.10855978

$-0.51002862$

$-1.40614111$

$-0.51000777$

1.10861982

1. 40598631

0.51017970

$-1.10850941$

$-1.40594206$

1.40611051

0.50986034

$-1.10867103$

\section{Coordinates of [3]-ladderene hydrocarbon}

$\mathrm{x}$

$\begin{array}{lr}\text { C } & 0.41528929 \\ C & 0.41528929 \\ C & -0.40545977 \\ C & -0.40545977\end{array}$

Y

2.00981049

2.00981049

0.73334474

0.73334474
0.67425724

$-0.67425724$

0.80303412

$-0.80303412$ 


$\begin{array}{lrrr}\mathrm{C} & 0.45144758 & -0.57496635 & 0.79582986 \\ \mathrm{C} & -0.40545977 & -1.87942101 & 0.78150917 \\ \mathrm{C} & 0.45144758 & -0.57496635 & -0.79582986 \\ \mathrm{C} & -0.40545977 & -1.87942101 & -0.78150917 \\ \mathrm{H} & 0.89947575 & 2.62983367 & 1.41695902 \\ \mathrm{H} & 0.89947575 & 2.62983367 & -1.41695902 \\ \mathrm{H} & -1.30984497 & 0.74001290 & 1.40880391 \\ \mathrm{H} & -1.30984497 & 0.74001290 & -1.40880391 \\ \mathrm{H} & 1.35208216 & -0.57629202 & 1.40586395 \\ \mathrm{H} & 0.10635223 & -2.73819881 & 1.21649090 \\ \mathrm{H} & -1.38296916 & -1.78796299 & 1.25830540 \\ \mathrm{H} & 1.35208216 & -0.57629202 & -1.40586395 \\ \mathrm{H} & 0.10635223 & -2.73819881 & -1.21649090 \\ \mathrm{H} & -1.38296916 & -1.78796299 & -1.25830540\end{array}$

\section{Coordinates of [3]-ladderdiene hydrocarbon}

\begin{tabular}{ccrr} 
& $x$ & $y$ & \multicolumn{1}{c}{$z$} \\
C & & & \\
C & 0.72072117 & 1.83563742 & 0.67322441 \\
C & 0.72072117 & 1.83563742 & -0.67322441 \\
C & 0.72072117 & 0.31341750 & 0.80032104 \\
C & 0.72072117 & 0.31341750 & -0.80032104 \\
C & -0.72072117 & -0.31341750 & 0.80032104 \\
C & -0.72072117 & -1.83563742 & 0.67322441 \\
C & -0.72072117 & -0.31341750 & -0.80032104 \\
H & -0.72072117 & -1.83563742 & -0.67322441 \\
H & 0.66025041 & 2.61748162 & 1.41797960 \\
H & 0.66025041 & 2.61748162 & -1.41797960 \\
H & 1.48439854 & -0.17210371 & 1.40280502 \\
H & 1.48439854 & -0.17210371 & -1.40280502 \\
H & -1.48439854 & 0.17210371 & 1.40280502 \\
H & -0.66025041 & -2.61748162 & 1.41797960 \\
H & -1.48439854 & 0.17210371 & -1.40280502 \\
& -0.66025041 & -2.61748162 & -1.41797960
\end{tabular}

